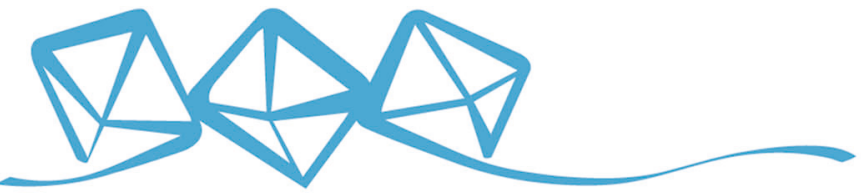 COMMUNICATIONS MATERIALS
}

ARTICLE

https://doi.org/10.1038/s43246-020-0020-7

OPEN

\section{Design principles for efficient photoelectrodes in solar rechargeable redox flow cell applications}

\author{
Dowon Bae (10 ${ }^{1,5 凶}$, Gerasimos Kanellos ${ }^{1,5}$, Gerrit M. Faasse ${ }^{1}$, Emil Dražević ${ }^{2}$, Anirudh Venugopal ${ }^{1}$ \& \\ Wilson A. Smith (10 $1,3,4 \bowtie$
}

Recent advances in photoelectrochemical redox flow cells, such as solar redox flow batteries, have received much attention as an alternative integrated technology for simultaneous conversion and storage of solar energy. Theoretically, it has been reported that even singlephoton devices can demonstrate unbiased photo-charging with high solar-to-chemical conversion efficiency; however, the poor redox kinetics of photoelectrodes reported thus far severely limit the photo-charging performance. Here, we report a band alignment design and propose surface coverage control to reduce the charge extraction barrier and create a facile carrier pathway from both $\mathrm{n}$ - and $\mathrm{p}$-type photoelectrodes to the electrolyte with the respective redox reaction. Based on these observations, we develop a single-photon photocharging device with a solar-to-chemical conversion efficiency over $9.4 \%$ for a redox flow cell system. Along with these findings, we provide design principles for simultaneous optimisation, which may lead to enhanced conversion efficiency in the further development of solarrechargeable redox flow cells.

\footnotetext{
${ }^{1}$ Delft University of Technology, Department of Chemical Engineering - Materials for Energy Conversion and Storage (MECS), Delft 2600GA, The Netherlands. ${ }^{2}$ Department of Engineering - Aarhus University, Aabogade 40, DK-8200 Aarhus, Denmark. ${ }^{3}$ National Renewable Energy Laboratory (NREL), Golden, CO, USA. ${ }^{4}$ Renewable \& Sustainable Energy Institute, University of Colorado Boulder, Boulder, CO, USA. ${ }^{5}$ These authors contributed equally: Dowon Bae, Gerasimos Kanellos. ${ }_{\text {eemail: d.bae@tudelft.nl; w.smith@tudelft.nl }}$
} 
$\mathrm{T}$ he increasing demand for integration of renewable energies into the grid calls for an energy storage system (ESS) that can provide a safe and effective solution to the inherent nature of temporal intermittencies, like sunlight. In this context, the utilisation of solar energy through photoelectrochemical (PEC) processes-including solar water splitting ${ }^{1,2}$ and other types of solar fuel $\left(\mathrm{CO}_{2} \text { or } \mathrm{N}_{2} \text { reduction }\right)^{3,4}$-has been regarded as being particularly attractive for storing solar energy. However, the sluggish kinetics (e.g. large overpotential or poor selectivity) and cost of electrification of the stored energy (e.g. fuel cells) impede the wide implementation of these PEC fuel systems ${ }^{5,6}$. Another approach that has received attention for solar energy storage is integration with secondary batteries ${ }^{6,7}$. To date, Li-ion solid-state batteries are the prime concern regarding commercially available ESS, owing to its high energy density and durability. Despite these advantages, recent fire incidents in various countries highlight the dangers associated with thermal runaway of lithium-based batteries in an ESS setting ${ }^{8,9}$. Meanwhile, redox cells, such as redox flow batteries (RFBs) generally allow safer servicing-for example, $0 \mathrm{~V}$ discharging-which is not possible in conventional Li-ion batteries without permanent damage. In addition, a catastrophic failure of RFBs would not likely cause a fire with associated toxic gases that occur in Li-ion failures ${ }^{10}$. Also, independence of energy capacity and power rate in RFBs is one of the main advantages 11,12 .

Even though the initial solar redox storage cell concept was demonstrated in 1976 by Hodes et al. ${ }^{13}$, most demonstrations have been reported rather recently ${ }^{14,15}$ in the wake of PEC materials which have been utilised for solar-fuel production ${ }^{1,2}$. Unlike conventional PEC fuel conversion processes, SRFB offers flexibility with respect to redox potential, which results in an unprecedented wide selection of band-gap $\left(\mathrm{E}_{\mathrm{g}}\right)$ of PEC materials. McKone et al. ${ }^{6}$ and other researchers ${ }^{16,17}$ have shown meaningful conversion efficiencies using small band-gap semiconductors, such as c-Si $(1.12 \mathrm{eV})$ and c-WSe $2(1.2 \mathrm{eV})$. Further, general charging/ discharging reactions in RFB present facile kinetics, which can be several orders of magnitudes faster than other electrolysis technologies that require high overpotential ${ }^{18}$. Despite these advantages of redox batteries, SRFB studies are confronted with slow kinetics at the photoelectrode surface, which is opposite to the trend in RFB studies. Wedege et al. ${ }^{16}$ demonstrated a c-Si photocharging device with the $\mathrm{Pt}$ conducting layer showing an overpotential of $\sim 400 \mathrm{mV}$ at $10 \mathrm{~mA} \mathrm{~cm}^{-2}$ for reduction of $\mathrm{Fe}$ $(\mathrm{CN})_{6}{ }^{3-}$ in $\mathrm{NH}_{4} \mathrm{Cl}$ electrolyte, whereas only approximately $130 \mathrm{mV}$ was required for water reduction at the same $\mathrm{pH}$ using the same device. Cheng et al. ${ }^{19}$ also reported a dual-bed type SRFB using a combination of $\mathrm{Ta}_{3} \mathrm{~N}_{5}$ and Si-based devices, which exhibited overpotentials of approximately $300 \mathrm{mV}$ and $400 \mathrm{mV}$, respectively. Most recently, Urbain et al. and Wenjie et al. reported outstanding double-digit STC\% (12.3 and 14.1\%, respectively) using monolithic tandem a-Si and III-V semiconductor-based devices ${ }^{15,20}$.

A common feature from those reports is the application of device architectures identified by photocatalytic water splitting researches. Nevertheless, the unforeseen poor reaction kinetics are likely attributed to an inadvertent application without careful consideration of solid/liquid interfaces, which may be different from typical catalytic reactions or charging/discharging in RFBs. The effect of this poor kinetics can be remedied by using a highcell-voltage multi-junction device approach, as mentioned above. However, the critical challenge is the demonstration of a top cell with a cost below $\$ 100$ per $\mathrm{m}^{2}$ and an efficiency above $20 \%$, which is essential for having a lower LCOE (Levelized Cost of Electricity) than a single-junction photon-device system ${ }^{21}$.

Therefore, there is a need to understand the fundamental aspects of interfaces between the photoelectrode and the electrolyte, as they pertain both to electric conductivity and energy level matching of the surface, for maximization of photocharging efficiency (i.e. solar-to-chemical efficiency, STC\%). Herein, we show how the charge transfer at the interface in SRFBs is different from that of the solar water splitting cases and present what we believe is the first report on conducting layer coverage effect and energy barrier scaling of the overpotential in redox reaction kinetics and, consequently, current-voltage behaviour. Using a c-Si photoelectrode with a buried pn-junction, which enables back-side light illumination without parasitic optical $\operatorname{loss}^{17}$, we demonstrate a dependency of ferri-/ferrocyanide redox reaction reactivity on the type of conducting materials and achieve the highest photo-charging efficiency among solarrechargeable redox flow cells with the single photoabsorber device. Finally, we show how these observations suggest general design principles for maximizing the photo-charging efficiency in any solar-rechargeable redox flow cells and RFBs with the direct immersion of the device.

\section{Results}

Reactivity trend in dark electrochemical reaction. Electrochemical linear sweep voltammogram (LSV) measurements were conducted (Fig. 1) in order to study the electrochemical reduction reactivity of the various conducting materials sputtered on highly doped silicon (c-Si) substrate (see inset). For a few samples, the $\mathrm{TiO}_{2}$ protection layer was sputtered as described elsewhere ${ }^{1,14}$ prior to the conducting layer deposition (Fig. 1b). In present work, Pt and carbon are chosen as conducting materials since those have proven to be effective on the photoelectrode surface for the redox reaction 16,20,22,23. Particularly, the carbon is favourable for the redox chemical cells since the noble metals, including Pt, can lead to an unexpected catalytic reaction, such as hydrogen evolution (HER), which can build an internal gas pressure and, consequently, potential mechanical damage on the system. As shown in Fig. 1a, good performances with high onset potentials $\left(V_{\text {on }}\right.$ at $\left.10 \mathrm{~mA} \mathrm{~cm}^{-2}\right)$ were observed for the asdeposited $\mathrm{Pt}$ and carbon films (black and blue) during the cathodic reduction of $\mathrm{Fe}(\mathrm{CN})_{6}{ }^{3-}$ (i.e. $0 \%$ state-of-charge (SOC)) in $1 \mathrm{M} \mathrm{NH}_{4} \mathrm{Cl}$. The samples exhibit similar trends under anodic reaction conditions (see Pt and carbon in 0, 50, and 100\% SOC in Supplementary Fig. 1 and Supplementary Note 1), thereby indicating that this is not related to the chemical affinity difference between the catholyte and anolyte. It has been accepted that conventional redox reactions in RFB are non-selective with respect to the electrode material ${ }^{22}$ unless intermediate catalytic reactions take place, such as metal hydride in the acidic supporting electrolyte ${ }^{24}$. Despite the fact that the redox reaction of the ferri-/ferrocyanide involves a simple single-electron transfer reaction, an apparent material dependency was observed for both cases (with and without protection layer). Poor reactivity of the bare c-Si sample (Fig. 1a, grey) can be attributed to a deactivation of the surface by oxidation or silanol group formation, as confirmed by previous studies ${ }^{2,24}$. Interestingly, while the sample with $\mathrm{Pt}$ and $\mathrm{TiO}_{2}$ interlayer showed almost unchanged results compared to the result from the sample without the $\mathrm{TiO}_{2}$ in Fig. 1a, the combination of the carbon with the $\mathrm{TiO}_{2}$ resulted in a substantial negative shift in $V_{\text {on }}(\sim 0.9 \mathrm{~V})$. Further, the $\mathrm{TiO}_{2}$ film with co-catalysts showed an efficient charge transfer performance, which is proven for water reduction studies ${ }^{1,16}$; thus, this unexpected poor activity in ferricyanide reduction cannot be explained by a conductivity investigation.

It is useful to perform a Mott-Schottky plot analysis (see Supplementary Fig. 2) to determine any impacts that these conducting layers may have on the band structure (e.g. due to Fermi level shift). We note that the Mott-Schottky analysis for 

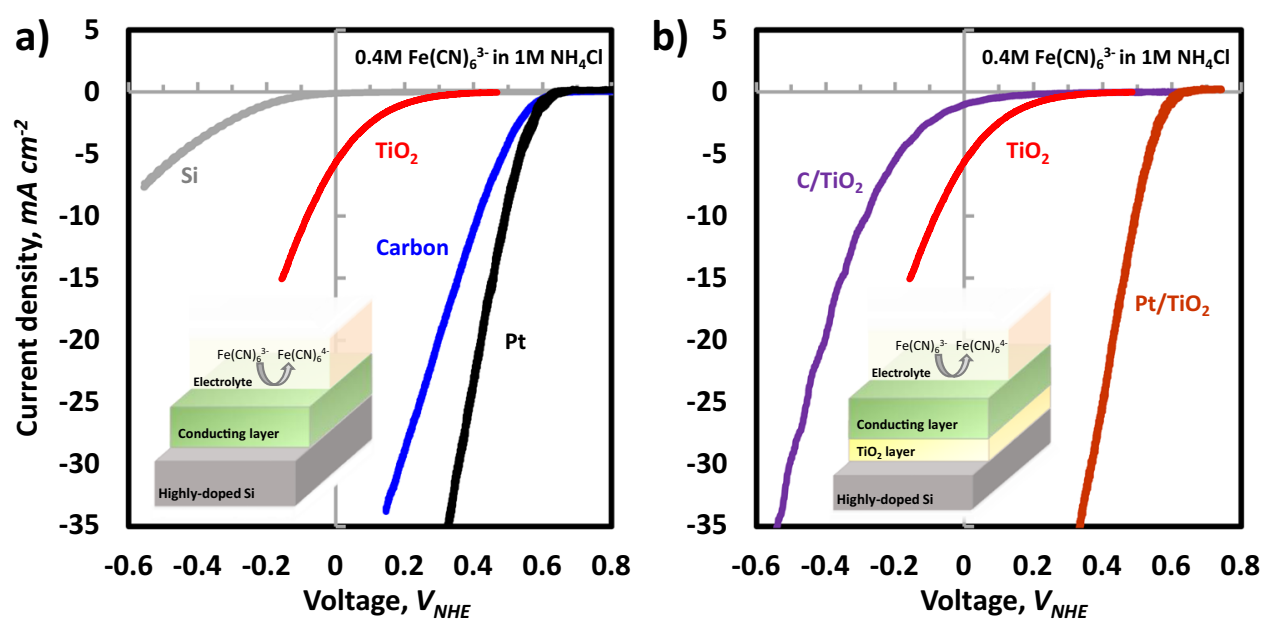

Fig. 1 Electrochemical LSV results for various conducting layers. Conducting materials deposited directly on the degenerately doped $\mathrm{Si}$ (a) and samples with $\mathrm{TiO}_{2}$ interlayer deposited on the same substrate (b). Both measurements were conducted in $0.4 \mathrm{M} \mathrm{Fe}(\mathrm{CN})_{6}{ }^{3-}$, with $1 \mathrm{M} \mathrm{NH}_{4} \mathrm{Cl}$ supporting electrolyte at $\mathrm{pH} 7$ (adjusted by using $\mathrm{NH}_{4} \mathrm{OH}$ ).

a)

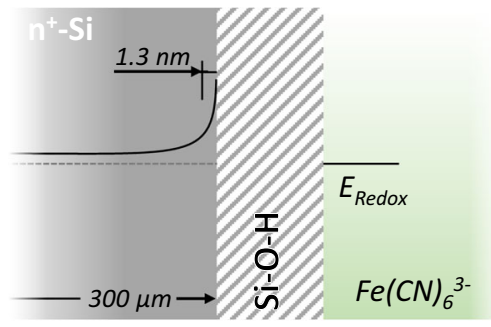

c)

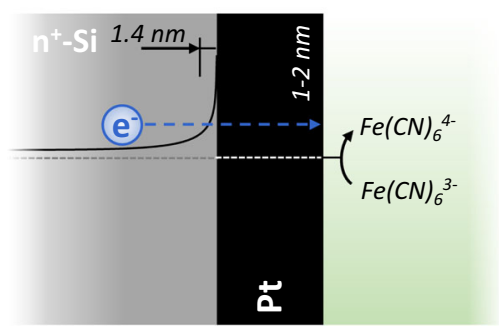

b)

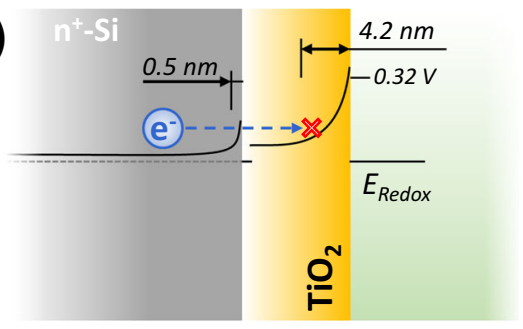

d)

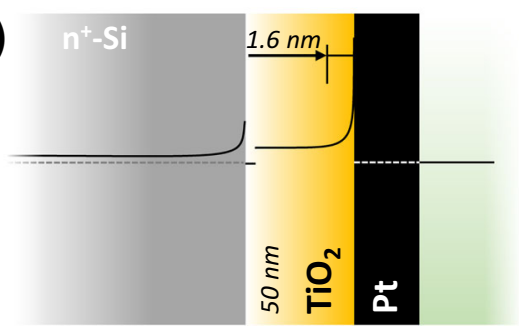

Fig. 2 Energy diagrams and charge transfer. Band alignments in the electrolyte for the bare c-Si electrode (a), $\mathrm{TiO}_{2}(\mathbf{b}), \mathrm{Pt}(\mathbf{c})$, and $\mathrm{Pt} / \mathrm{TiO} \mathrm{O}_{2}(\mathbf{d}) . \mathrm{Note}$ that barrier heights are marked with respect to the redox potential.

conducting materials of Pt and carbon is invalid since no space charge layer is formed. In the bare Si case, obtaining a reliable result is rather challenging due to surface deactivation caused by silane or oxide formation in the electrolyte (Fig. 2a), as described in previous studies ${ }^{1,2}$. It is noteworthy that the surface of the $\mathrm{Si}$ was chemically cleaned upon any electrochemical analysis by using a $3 \mathrm{M} \mathrm{H}_{2} \mathrm{SO}_{4}$ solution in order to remove the native oxide layer. Using a highly acidic supporting electrolyte, such as HF, can provide a reliable environment for the measurement ${ }^{25}$, but one cannot avoid the formation of a toxic $\mathrm{HCN}$ due to instability of the ferro-/ferricyanide in low $\mathrm{pH}$ conditions ${ }^{17}$. In the case of the semiconductor/liquid junctions, the flat band potential is found to be $0.32 \mathrm{~V}$ for the $\mathrm{TiO}_{2}$. These results imply that upward band bending within the solid phase at the equilibrium can form a Schottky barrier with a thick depletion width $(4.2 \mathrm{~nm}$, Fig. $2 \mathrm{~b})$, which electrons cannot tunnel through (detailed calculation can be found in Supplementary Note 2). In Fig. $2 c$ and d, the complete band diagram of the $\mathrm{Pt}$ conducting layer cases with and without the $\mathrm{TiO}_{2}$ protective layer are schematically depicted, assuming an ohmic-like contact with very narrow depletion width of 1.4 and $\sim 1.6 \mathrm{~nm}$ within the $\mathrm{n}^{+}-\mathrm{Si}$ and $\mathrm{TiO}_{2}$, respectively. These assemblies enable a feasible electron transfer throughout the conduction band of $\mathrm{TiO}_{2}$ and compact metal layer, as illustrated. These feasible charge transfers were supported by the steep slope of the $\mathrm{Pt}$ and $\mathrm{TiO}_{2} / \mathrm{Pt}$ samples with onset potential $\left(V_{\text {on }}\right)$ of $\sim 0.5$ $\mathrm{V}_{\mathrm{NHE}}$ in Fig. 1b. It is noteworthy that carbon with similar work function $(\sim 5 \mathrm{eV})$ is also expected to have a similar tunnelling barrier as the Si/Pt case depicted in Fig. 2c, e (see Supplementary Note 2).

An interesting feature is, as mentioned above, the conducting carbon deposited onto the $\mathrm{TiO}_{2}$. While the carbon film showed rather fast kinetics close to that from the $\mathrm{Pt}$ and $\mathrm{Pt} / \mathrm{TiO}_{2}, \mathrm{C} / \mathrm{TiO}_{2}$ combination showed poor kinetics with a negative shift of both $\mathrm{V}_{\mathrm{on}}$ and $\mathrm{E}_{\mathrm{FB}}$, which end up close to those from the $\mathrm{TiO}_{2}$. This can be attributed to poor coverage of the carbon. Figure 3a presents LSV curves obtained from the carbon deposited electrode with various deposition conditions. According to observation using quartz crystal microbalance $(\mathrm{QCM})$ monitoring, the baseline carbon deposited for $10 \mathrm{~min}$ is expected to have an average thickness of 1-2 nm, and the $60 \mathrm{~min}$ deposited sample has an 

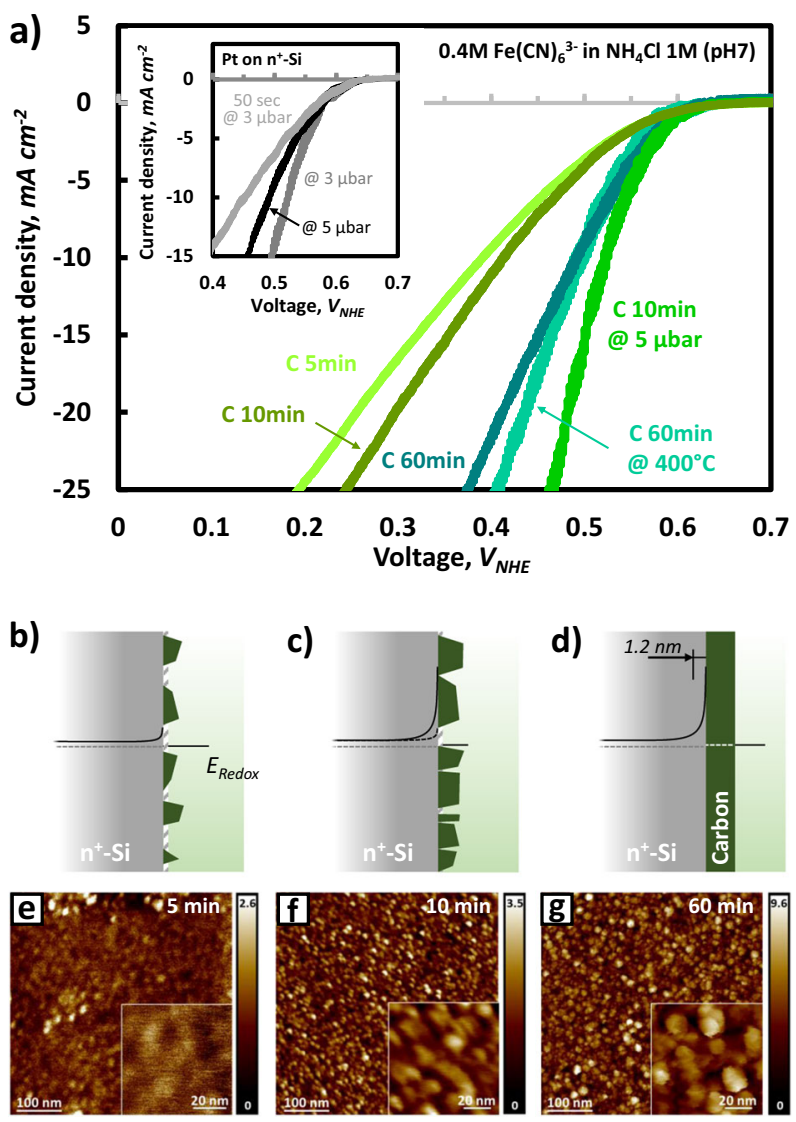

Fig. 3 LSVs of c-Si electrodes with carbon conducting layer. LSVs for the carbon layers deposited under various conditions (e.g. deposition duration and working pressure of the sputtering) (a). LSVs for the samples with Pt conducting layers are also demonstrated in the inset. For notational simplicity, 'min', 'sec', and ' $\mu$ bar' correspond to the deposition time and pressure of the sputtering chamber, respectively. The cross-sectional schematic of the electrode with various carbon coverages are also illustrated here (b-d). AFM images showing carbon islands distribution and size variation with deposition time $(\mathbf{e}-\mathbf{g})$.

average thickness of $6-8 \mathrm{~nm}$. On the assumption that the thickness of the film is proportional to the duration of the deposition, a noticeable enhancement of activities in LSV curves is mainly attributed to the improved coverage of the electrode surface and, consequently, minimised substrate oxidation upon direct contact with the electrolyte, as illustrated in Fig. 3b-d. Generally, working pressure during the sputter deposition is directly related to the porosity, or void density of the film ${ }^{26,27}$. As depicted in Fig. 3a, carbon electrodes deposited at lowered working pressure $(5 \mu \mathrm{bar})$ showed outstanding activity, surpassing the sample with unoptimized Pt film in Fig. 1a. In the case of the carbon-coated electrode annealed at $400^{\circ} \mathrm{C}$, the $J-V$ curve shows a slightly larger cathodic current than the curve with the same deposition time $(60 \mathrm{~min})$. This can be attributed to the reduced resistivity of carbon owing to the carrier mobility increased with increasing annealing temperature as reported previously ${ }^{28}$. Similarly, this approach is also applicable to the $\mathrm{Pt}$ case, as depicted in the inset of Fig. 3a. An electrode with the Pt deposited at lowered working pressure $(3 \mu \mathrm{bar})$ showed improved kinetics compared to the baseline sample used in Fig. 1a (5 $\mu \mathrm{bar}$, $90 \mathrm{~s}$ at room temperature). On the other hand, activity from the Pt electrode deposited within a shorter period $(50 \mathrm{~s}, \sim 3 \mathrm{~nm})$ decreased significantly; these results are in good agreement with the observation from the deposited carbon electrodes.
Simultaneously, it is also possible that the 'pinch-off effect is responsible for this observation. Considering an ohmic-like contact between the conducting layer and the substrate, the degree to which electrostatic screening of the substrate (i.e. pinchoff effect) influences the band diagram is linearly proportional to the depletion width within the silicon substrate 29,30 . The depletion width of the silicon substrate $(\sim 1.3 \mathrm{~nm}$ in Fig. 2a) implies that $\mathrm{Pt}$ or carbon islands with a diameter below the depletion width level are, to a large extent, electronically pinched off (detailed calculations are provided in the Supplementary Note 2) and, consequently, hinders a feasible charge transfer.

Further, the atomic force microscopy (AFM) analysis (Fig. $3 \mathrm{e}-\mathrm{g}$ ) demonstrates how the surface coverage by the carbon changes over the deposition time (also illustrated in Fig. 3b-d) and confirmed the correlation between the carbon coverage and duration of the deposition. The maximum height difference between the c-Si substrate and valleys of crests (i.e. carbon cluster with brighter colour) significantly changes from $2.6 \mathrm{~nm}$ to $9.6 \mathrm{~nm}$ for the sample with 60-min deposited carbon (Fig. 3g). The thickness changes also were also verified by the profilometer with a sub-nanometer vertical resolution. It is evident that the carbon deposition resulted in a rather well-distributed particle or island formation, which changes its size with deposition time. Naturally, only the sample with islands of sufficient size is expected to provide a facile carrier pathway over the pinch-off regime. What is clear though is this particle distribution control has been wellreported in photocatalysis community ${ }^{29,31}$, Nonetheless, the plain fact is that the in-depth analysis for the RFB implementation has not been studied well. Furthermore, unlike the photo- and electro-catalysis where the chemical selectivity takes place simultaneously, the redox reactions in RFB are obliged to depend heavily on the surface coverage rather than choosing a metal with high catalytic reactivity 22,23

Photoelectrochemical behaviour and efficiency loss analysis. Previously, we demonstrated functional redox flow batteries consisting of ferri-/ferrocyanide, coupled with TEMPO-sulphate and $\mathrm{NH}_{4} \mathrm{Br}^{16,17}$; thus, the same electrolyte conditions were used in this work. $\mathrm{Cu}^{2+1+}$ redox couples were additionally added as a means of obtaining a higher cell voltage than that with TEMPOsulphate. As evident from the cyclic voltammograms (CVs) using a Pt-coated degenerately doped c-Si electrode in Fig. $4 \mathrm{a}$, the redox couples are electrochemically reversible, and the redox potentials are as expected from previous reports $16,32,33$. These results lead to photo-charging with a cell potential of $0.34 \mathrm{~V}, 0.40 \mathrm{~V}$, and $0.65 \mathrm{~V}$ for the ferri-/ferrocyanide with TEMPO- and copper-sulfate, and $\mathrm{NH}_{4} \mathrm{Br}$, respectively.

Photo-charging tests were conducted with both three-electrode configuration for half-cell reactions (Fig. $4 \mathrm{~b}$ and d) and twoelectrode setup (see Supplementary Note 7 for more detail) with different electrolytes separated by the Nafion membrane (Fig. 4c and e). In the current study, the back-illuminated $\mathrm{pn}^{+}$and $\mathrm{np}^{+}-\mathrm{Si}$ photoelectrodes were used as a photo-charging device (see Table 1). Upon illumination, photo-excited electron and hole pairs enter the conduction and valence bands, respectively, and move as illustrated in the band diagram in Fig. $4 \mathrm{f}$ and $\mathrm{g}$ (also see Supplementary Fig. 3). These devices have been proven as effective photoelectrodes, which deliver photovoltage $\left(V_{p h}\right)$ of over $0.51 \mathrm{~V}$ in previous SRFB studies ${ }^{16,17}$. Onset potential (at $10 \mathrm{~mA} \mathrm{~cm}^{-2}$ ) under the light of approximately $0.8 \mathrm{~V}_{\mathrm{NHE}}$ from the LSVs under the light (Fig. 4b black and green) also support that the photoelectrode is functional with ferricyanide. We emphasise that we shall refrain from using the open circuit potential $\left(V_{o c}\right)$ since the net-zero current point remains ambiguous in the PEC device system $^{31}$. As demonstrated earlier in Figs. 2 and 3, 

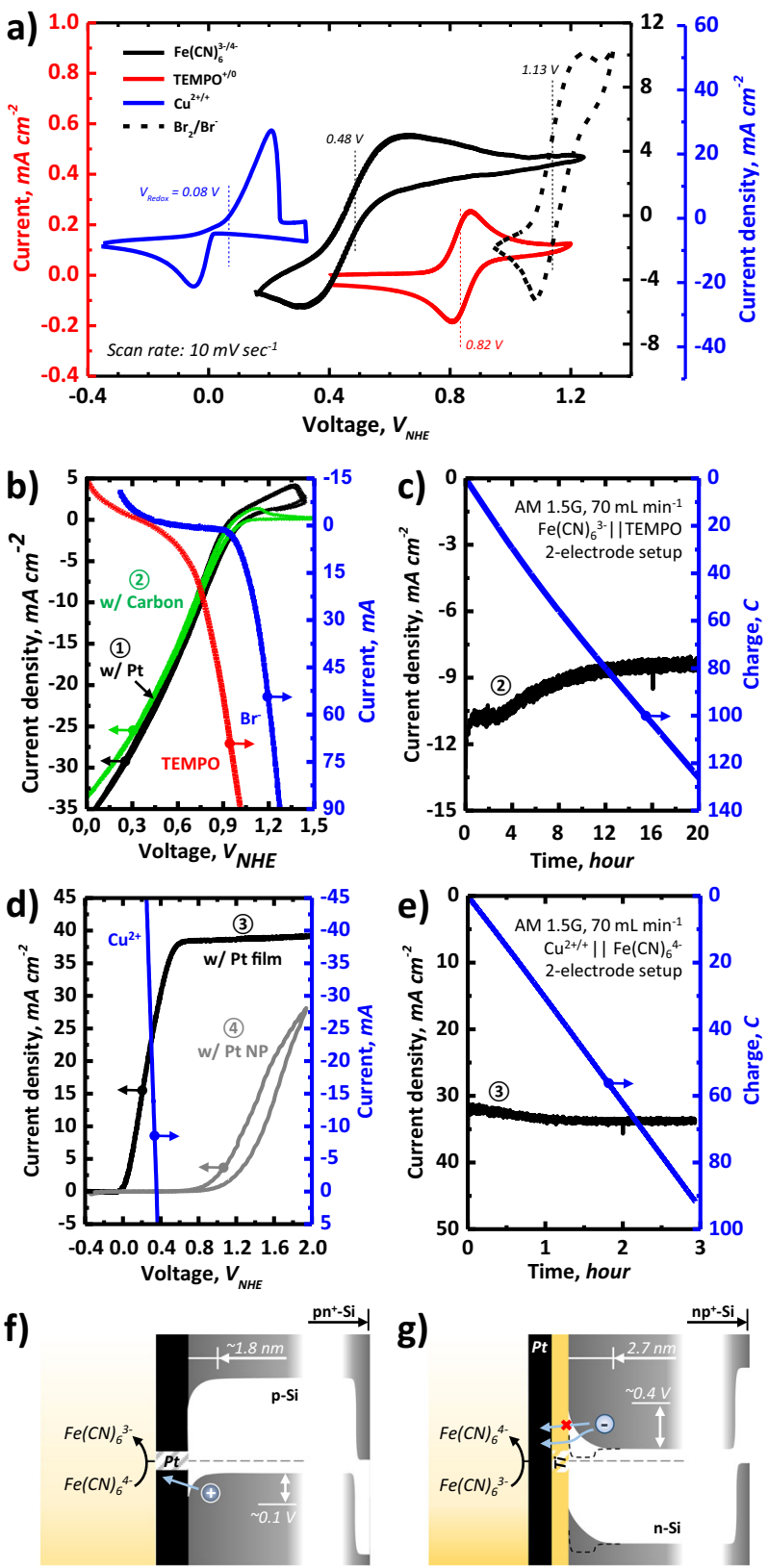

Fig. 4 CVs for redox couples and LSVs under illumination. a CVs of $2 \mathrm{mM}$ solutions of TEMPO-sulphate, $\mathrm{CuSO}_{4}, \mathrm{~K}_{3} \mathrm{Fe}(\mathrm{CN})_{6}$, and $\mathrm{NH}_{4} \mathrm{Br}$ using a scan rate of $10 \mathrm{mV} \mathrm{sec}^{-1}$. For the TEMPO-sulphate, $\mathrm{K}_{3} \mathrm{Fe}(\mathrm{CN})_{6}$ and $\mathrm{NH}_{4} \mathrm{Br}$, $\mathrm{NH}_{4} \mathrm{Cl}$ was used as a supporting electrolyte, and the $\mathrm{pH}$ was adjusted to 7 . The $\mathrm{pH}$ of the $\mathrm{Cu}^{2+}$ electrolyte was adjusted to 2 (with $\mathrm{H}_{2} \mathrm{SO}_{4}$ ). b Overlaid current-voltage curves measured in a three-electrode experiment with carbon felt as an anode. $\mathrm{C} / \mathrm{pn}^{+}-\mathrm{Si}$ and $\mathrm{Pt}(\mathrm{Ti}) / \mathrm{pn}^{+}-\mathrm{Si}$ in a $0.4 \mathrm{M} \mathrm{Fe}(\mathrm{CN})_{6}{ }^{3-}$; while carbon-felt anode in $0.4 \mathrm{M}$ of $\mathrm{TEMPO}^{-}$and $\mathrm{Br}^{-}$. c CA and accumulated charge of the photocathode with carbon layer under the same conditions, but with a two-electrode configuration. d Overlaid currentvoltage curves for the photoanodes. The $\mathrm{pn}^{+}$-Si photoanodes with a Pt thin film (5-7 nm) and Pt nanoparticles (200 $\mathrm{ng} \mathrm{cm}^{-2}$ ) tested in $0.4 \mathrm{M} \mathrm{Fe}$ $(\mathrm{CN})_{6}{ }^{4-}$ and carbon-felt cathode for $0.4 \mathrm{M} \mathrm{Cu}^{2+}$. e CA and accumulated charge of the photoanode under two-electrode configuration. Schematic energy diagrams for both $\mathrm{p}$-type (f) and $\mathrm{n}$-type surface samples $(\mathbf{g})$ are also calculated. Note that numbers with circle correspond to the sample number in Table 1.
Table 1 Photoelectrode designa and function.

\begin{tabular}{lll} 
Sample No. & Configuration & Function \\
\hline 1 & $\mathrm{Pt} / \mathrm{Ti} / \mathrm{np}^{+}-\mathrm{Si} / \mathrm{Au}$ (light) & Photocathode \\
2 & $\mathrm{C} / \mathrm{Ti} / \mathrm{np}^{+}-\mathrm{Si} / \mathrm{Au}$ (light) & Photocathode \\
3 & $\mathrm{Pt} / \mathrm{pn}^{+}-\mathrm{Si} / \mathrm{Au}$ (light) & Photoanode \\
4 & $\mathrm{Pt} \mathrm{NP} / \mathrm{np}^{+}-\mathrm{Si} / \mathrm{Au}$ (light) & Photoanode \\
\hline aThe light illumination side is marked with (light). & \\
\hline
\end{tabular}

photocathodes with both Pt and carbon show rather similar LSV behaviour when they are coupled with photocathode under light illumination. It is noteworthy that replacing the Pt to the carbon is important for redox battery applications due to outstanding kinetics of hydrogen evolution reaction with Pt. At a $0 \%$ SOC condition (i.e. before photo-charging), a photocurrent of around $35 \mathrm{~mA} \mathrm{~cm}^{-2}$ is reached. Compared to the result under the same conditions $\left(\sim 11 \mathrm{~mA} \mathrm{~cm}^{-2}\right)$ in our previous report, this high photocurrent probably is attributed to the direct light absorption without any parasitic optical loss by the electrolyte and other layers including protective layer, Pt conducting layers, and acrylic window, which are unavoidable in front-side illumination conditions $^{16}$. We note that the cross-point of current-voltage curves of the photocathode and anode in Fig. $4 \mathrm{~b}$ and d does not represent the operating current. The unit of the $y$-axis is current, not current density, since the anode is composed of thick carbonfelt whose geometrical area is hard to be measured. Instead, the current densities were determined using an electrochemically active surface area (ECSA; see also Supplementary Note 8) and projected area $\left(2 \mathrm{~cm}^{2}\right)$ of the carbon-felt, and the LSVs are plotted in Supplementary Fig. 4.

Figure $4 \mathrm{c}$ illustrates the photo-charge profiles for the first $20 \mathrm{~h}$ using a $\mathrm{C} / \mathrm{np}^{+}-\mathrm{Si} \|$ Carbon-rod configuration with ferricyanide $\|$ TEMPO-sulphate (2-electrode configuration; see Supplementary Fig. 5). The charging photocurrent for the photocathode begins at $11.6 \mathrm{~mA} \mathrm{~cm}^{-2}$ and decreases to $8.7 \mathrm{~mA} \mathrm{~cm}^{-2}$; the corresponding initial and final values for the ferricyanide $\mid \mathrm{Br}_{2} / \mathrm{Br}^{-}$cell are 4.7 and $0.4 \mathrm{~mA} \mathrm{~cm}^{-2}$ (see Supplementary Fig. 5). The difference in photocurrent density between the two setups is due to a redox potential shift leading to an increase in the cell voltage (from 0.34 to $0.62 \mathrm{~V}$ ) that lowers the operating current density (intersection between the polarisation curves in Fig. $4 \mathrm{~b}$ ). Theoretically, $0.51 \mathrm{~V}$ from the photocathode is insufficient to charge the ferricyanide\| $\mathrm{Br}_{2} / \mathrm{Br}^{-}$battery without a bias potential. The low photo-charging current from the ferricyanide || $\mathrm{Br}_{2} / \mathrm{Br}^{-}$cell is only observable at low SOC\% where the required potential is quite low according to the Nernst equation ${ }^{14,17}$. Photo-charging with a photoanode sample with Pt conducting film (black curve) showed similar photocurrent output, as it showed as a photocathode in Fig. 4c. Further, the slope near the $V_{o c}$ region exhibited a relatively sharp increase, thereby indicating more facile electrode kinetics and charge carrier transport. Actually, both ferri- and ferrocyanide are the most commonly used electron and hole scavenger, respectively, with similar reaction rates. What is of importance here is that both electrodes have the same conducting layer $(\mathrm{Pt})$ but substrates with different doping type and concentration. Electron or hole transfer from the c-Si to the Pt would most probably have to occur through tunneling, which is limited by WentzelKramers-Brillouin (WKB) approximation, where the doping density and effective mass can consequently have a significant impact on both triangular tunnel barrier and current limit ${ }^{29}$. Using the WKB approximation, the Pt/p-Si junction for the photoanode structure appears to have a negligible ohmic-like junction (Fig. 4f), whereas $\mathrm{Pt}(\mathrm{Ti}) / \mathrm{n}$-Si junction for the photocathode has a triangular tunnel barrier of almost 2-3 nm (Fig. 4g), 
thereby resulting in a current limit that is 10 times lower than the one with Pt/p-Si (see Supplementary Note 2).

Notwithstanding the facile kinetics in the photoanode case, Fig. 4d reveals an interesting feature. A photoanode with $\mathrm{Pt}$ nanoparticles with $200 \mathrm{ng} \mathrm{cm}^{-2}$ loading was produced using the procedure described in our previous work ${ }^{1,34}$. The LSV curve of the sample with Pt nanoparticles (grey) is described accurately at low $V_{\text {on }}$ with slow kinetics and low current density. Considering that the physical surface coverage by the nanoparticles is less than $5 \% 35$, it is reasonable that deactivated substrates without conducting particles can hinder facile carrier transport as illustrated in Supplementary Fig. 3. This result is in good agreement with the sample that has carbon coverage, as demonstrated earlier in Fig. 3. Note that both Pt film and nanoparticles were formed after the surface cleaning step (see Supplementary Note 4) to prevent the deactivation of the interface between the Si substrate and the conducting material.

Comparison of the simulated and experimental results. In Fig. 5a, modeled curves overlap with the experimental solar conversion efficiencies 3.0 and $9.44 \%$ at $10 \%$ SOC for the unbiased ferricyanide, TEMPO-sulphate and $\mathrm{Cu}$-sulphate, and ferrocyanide photo-charging cases, respectively. It must be noted that theoretical modeling was conducted using the same procedure derived from the Shockley and Queisser limitation ${ }^{36}$ previously reported by our group (also see Supplementary Note 6) ${ }^{17}$. It is also worthwhile to note that the conversion efficiencies were calculated using a cell voltage at given SOC, as shown in our previous reports ${ }^{16,17}$. This photoelectrochemical charging efficiency for the $\mathrm{Cu}$-sulphate||ferrocyanide cell is the highest among all the SRFBs with a single-photon device reported thus far. Naturally, the ferricyanide $\| \mathrm{Br}_{2} / \mathrm{Br}^{-}$combination case only showed $0.32 \%$ at $1 \%$ SOC due to the discrepancy between the photovoltage $(\sim 0.51 \mathrm{~V})$ and cell voltage $(0.65 \mathrm{~V})$ to drive the chemical reaction. A meaningful photocurrent output was only observable at the beginning of the long-term photo-charging experiment (Supplementary Fig. 6; see also Supplementary Note 3).

Comparison of the experimentally measured results and theoretical estimation provided in Fig. 5a reveals that the experimental results follow the predicted trend and that the overlap may serve as a guideline for further improvement. The discrepancy between the theoretical maximum at given cell potential indicates that there remains substantial room for improvement. Of particular significance is that to maximize the overall converted energy amount, the SOC-dependent efficiency limit shift must be taken into account in order to secure the high averaged STC\% (see Supplementary Note 6 for calculation details) over the entire charging cycle, as discussed in a previous report $^{17}$. According to theoretical estimation (Fig. 5d), the SRFB system with ferri-/ferrocyanide and copper, which exhibits cell voltage of $0.4 \mathrm{~V}$, can have a rather high conversion efficiency limit of over $10.9 \%$. However, on the basis of the SOC-dependent modeling, SRFB with a lower cell voltage (i.e. $0.3 \mathrm{~V}$ ) yields $\sim 30 \%$ more power conversion (i.e. integrated area under the curve) than the case with a cell voltage of $0.4 \mathrm{~V}$. Using redox couples with high-cell voltage is energetically favourable for achieving a high STC\% at low SOC\%, but it diminishes drastically with an increase in SOC\% (Supplementary Fig. 7). Optimisation of the cell voltage for maximizing the averaged STC\% over the entire cycle is beyond the scope of this paper; however, it must be addressed in further development. Moreover, a detailed investigation in this regard is underway.

Concerning the device configuration, a combination of the $J-V$ behaviour from the LSV in Fig. $4 \mathrm{~b}$ (corresponding to the measured
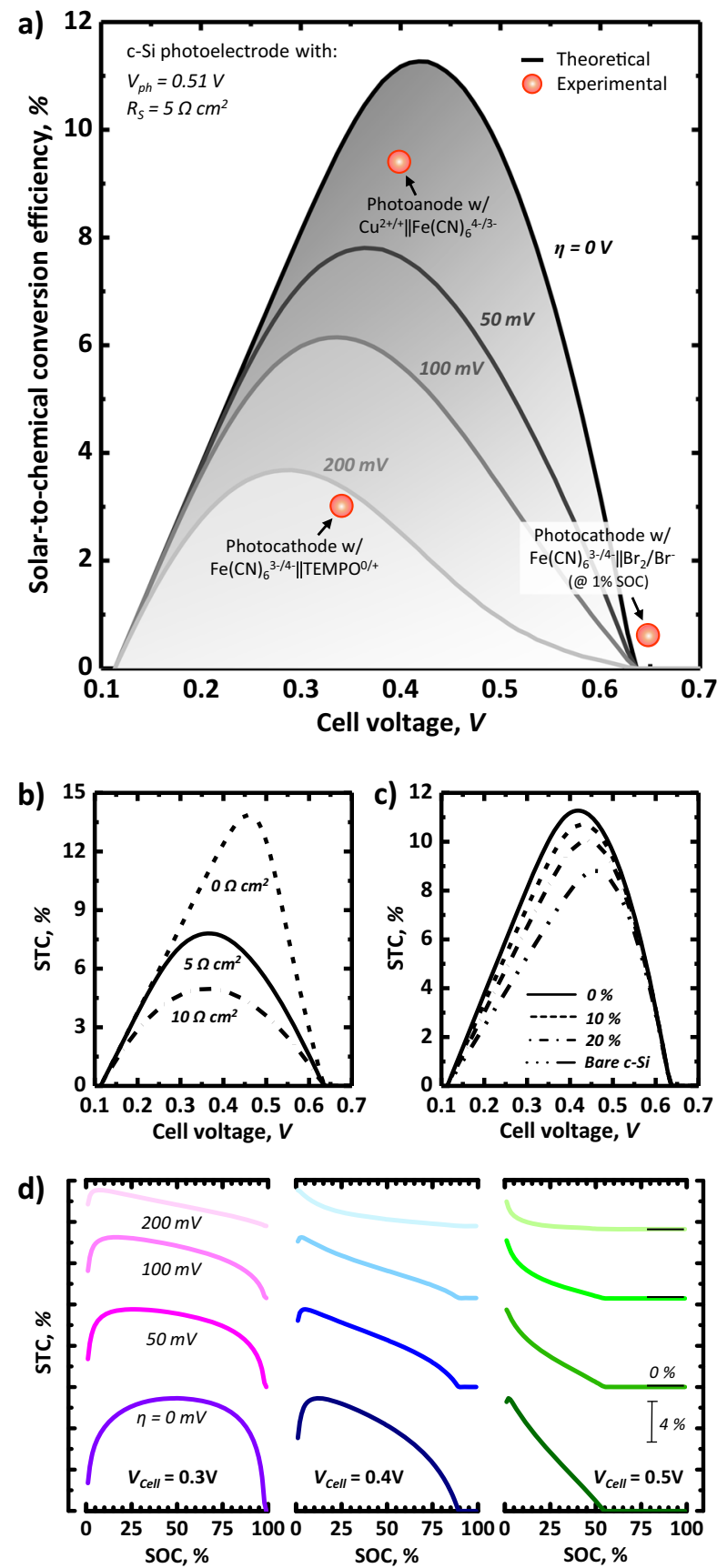

Fig. 5 Theoretical STC\% limits with experimental values. a The photocharging efficiency at $10 \%$ SOC is plotted as a function of the thermodynamic potential for different kinetic overpotentials $(\eta=0 \mathrm{~V}$, $50 \mathrm{mV}, 0.1 \mathrm{~V}, 0.2 \mathrm{~V}$ ). The system is modeled as a c-Si photoelectrode with a photovoltage $\left(V_{p h}\right)$ of $0.51 \mathrm{~V}$ and a series resistance $\left(R_{S}\right)$ of $5 \Omega \mathrm{cm}^{2}$. The red circles indicate the experimentally measured value with various redox couples from Fig. 4 b, c correspond to the model curves for different $R_{S}$ and reflection from the surface, respectively, and $\mathbf{d}$ demonstrates SOCdependant STC\% with varied cell voltage and overpotential.

data points at $0.34 \mathrm{~V}$ in Fig. 5a) and theoretical estimation engenders the rational inference that reducing the overpotential is vital for increasing the operating current density and efficiency. Unlike the photoanode sample with facile carrier transport across the $\mathrm{Pt} / \mathrm{p}-\mathrm{Si}$ junction (Fig. 4f), the photocathode with n-type Si surface requires almost $300 \mathrm{mV}$ with respect to onset potential; this experimental observation roughly agrees with the modeled estimation in Fig. 5a. Further, replacing the Ti interlayer 
with another metal with lower work function-for example, $\mathrm{Mn}$ $(4.1 \mathrm{~V})$ - or forming a conducting layer with a heavily doped sheet $\left(\mathrm{n}^{+}\right.$in this case) can reduce the Schottky barrier, as illustrated with a dashed line in Fig. 4g. The metal-oxide-semiconductor (MOS) configuration also enables efficient hole-transport from the n-type $\mathrm{Si}$ to the metallic $\mathrm{Pt}^{37}$. In contrast, in the case of the photoanode at $0.4 \mathrm{~V}_{\text {cell }}$ in Fig. $5 \mathrm{a}$, a different approach is required, since the $\mathrm{Pt} / \mathrm{pn}^{+}$-Si photoanode sample displayed fast kinetics $(\sim 100 \mathrm{mV}$ is required to obtain $10 \mathrm{~mA} \mathrm{~cm}{ }^{-2}$ ). An additional charge transport layer, such as highly doped n-type TCO, including indium tin oxide (ITO), can be considered as an interlayer between the $\mathrm{n}^{+}$-doped $\mathrm{Si}$ surface and metallic grid $(\mathrm{Cu} / \mathrm{Au}$ in this study) that enhances lateral conductivity.

As demonstrated in our previous studies ${ }^{16,33}$, the RFBs with ferricyanide, bromine, and TEMPO-sulphate used in this work are functional (also illustrated in Supplementary Fig. 8); however, discharging the RFB cell with a $\mathrm{Cu}^{2+/+}$ anolyte was chalenged by unexpected precipitation of a brownish compound at membrane, likely oxidised copper that is known to be accompanied by the oxidation process of copper ions ${ }^{32}$. This may be attributed to the crossover of protons through the Nafion membrane from the catholyte to anolyte side due to the $\mathrm{pH}$ difference between the chambers. Based on the Pourbaix diagram ${ }^{38}$, the copper ions tend to form precipitates of hydroxide/oxide at $\mathrm{pH}$ above 4 . Making a battery with copper ammonia complexes, which is stable in high $\mathrm{pH}$ range, would alleviate this instability. Replacing the Nafion by the bipolar membrane can be an alternative approach since it allows the use of different $\mathrm{pH}$ for each chamber ${ }^{39}$. Note that within the limited scope of this study, no modification or optimization work of the RFB cell has been elaborated; however, it should be addressed in further development.

It is evident from the advances in the field during the past few years that the envisioned realisation of the practical solar RFBs remains challenged by issues related to modest solar charging efficiency, mainly due to slow redox kinetics at the photoelectrode/liquid interface. In this sense, as shown in the present work, it is important to design the conducting layer carefully such that it is energetically well-matched to enable a feasible carrier transport from the photoelectrodes. Regardless of the recent monumental findings allowing high STC\% using a multi-junction device ${ }^{15,20}$, the interface energy barrier design, has not been well identified and optimized for redox cell applications, whereas it has been well-studied in PEC water splitting community ${ }^{31,40}$. Specifically, this puts emphasis that choosing an appropriate conductivity type of the electrode surface upon combination with high work function conductors, such as $\mathrm{Pt}$ and $\mathrm{C}$, is of great importance for minimizing the reaction overpotential. From the opposite point of view, this implies that there is significant room for improvement in the current-voltage performance as suggested by the theoretical modeling. However, there are still other operating parameters, like the volumetric loading effect on photo-charging performance (Supplementary Fig. 6), which must be addressed for further development. For instance, the long-term photo-charging experiment of the photoanode used in Fig. $4 \mathrm{~d}$ with a $15 \mathrm{~mL}$ electrolyte $(10 \mathrm{~mL}$ less than the volume used in Fig. $4 \mathrm{~d})$ showed an STC\% trend which appears to lag behind by $1.3 \%$ point. The STC\% trends for other samples with respect to the SOC\% change (i.e. light illumination time) can be found in Supplementary Fig. 9. In terms of stability, no significant damage to the photoelectrodes was observed. However, applying the passivation layer, such as $\mathrm{TiO}_{2}$, is also considerable for the practical application since the facile charge transfer across the $\mathrm{TiO}_{2}$ has been evidenced in Figs. 1 and 2.

In the overall device design-wise approach, an important limitation that must be overcome is the series resistance of the device. As demonstrated in Fig. 5b, similarly to the kinetic overpotential effect, obtainable maximum STC\% increases with decreasing series resistance, simultaneously shifting optimum cell potential shifts to higher values. Apart from the solid/liquid interface optimisation, the optimisation of the semiconductor/ metal interface is also required to reduce the overall series resistance. Interestingly, the strong reflection from the bare Si surface-which was inevitable for all samples tested in this study-contributes with a significantly lower impact on the STC\% change. As predicted in Fig. $5 \mathrm{c}$ and using the theoretical model from our previous report ${ }^{17}$, the c-Si modeled sample without any optical loss (i.e. 0\% reflection) shows an STC\% higher than 11\%; on the other hand, the sample with a bare Si surface exhibits an STC\% of approximately $8.7 \%$, with practical PV parameters used in Fig. 5a (i.e. $V_{p h}=0.51 \mathrm{~V}$ and $R_{S}=5 \Omega \mathrm{cm}$ ). Taking into account the fact that, in general, bare c-Si has a reflection of $\sim 30 \%$, this discrepancy between these two extreme conditions is not significant. In terms of the aspect of the redox system level, the increased solution conductivity is an obvious improvement that provides better kinetics. Thus, either using a highly concentrated electrolyte or conductive additives can be simple approaches ${ }^{17,41}$, but, at the same time, the corrosion of the device and unexpected by-products from the additives should be addressed.

\section{Discussion}

The importance of this work can be emphasised in terms of two aspects. First, the necessity of band alignment design in order to form a facile carrier pathway from both $\mathrm{n}$ - and $\mathrm{p}$-type photoelectrodes to the electrolyte with the respective redox reaction in the SRFB system is emphasised along with supporting experimental evidence. Specifically, the p-type surfac combined with high work function materials is appeared to be highly efficient for photoanodic-charging. A high solar-to-chemical conversion performance of over $9.4 \%$ can be achieved from a system with a cell voltage of $0.4 \mathrm{~V}$ using a photoanode with a $\mathrm{p}-\mathrm{Si} / \mathrm{Pt}$ interface. Although it must be emphasised that this addresses only "half of the SRFB challenge (i.e., photo-charging performance), to the best of our knowledge, this is the highest reported efficiency for the SRFB with a single-photon device system. We also found that the device performance is highly influenced by the surface coverage of the substrate by the conducting layer. Our findings suggest that minimising the deactivated surface is another key feature for facile carrier transfer. The comparison between the measured value and modeled curves identifies that there remains room to improve conversion efficiency. Further, in terms of the overall device-wise approach, various efforts-including optical loss management-must be made for further development.

\section{Methods}

Chemicals for electrolyte. The synthesis of TEMPO-4-sulfate was prepared from 4-Hydroxy-TEMPO (Henan Tianfu Chemical Co. LTD, $\geq 99 \%$ ); the detailed preparation procedure can be found in our previous publication ${ }^{16}$ (see also Supplementary Note 5). $\mathrm{K}_{3}\left[\mathrm{Fe}(\mathrm{CN})_{6}\right], \mathrm{K}_{4}\left[\mathrm{Fe}(\mathrm{CN})_{6}\right] \cdot 3 \mathrm{H}_{2} \mathrm{O}$ (Sigma-Aldrich, $\geq 99 \%$ ), and $\mathrm{NH}_{4} \mathrm{Br}$ (Sigma-Aldrich, $\geq 99.99 \%$ ) were used as received after mixing with $1 \mathrm{M}$ $\mathrm{NH}_{4} \mathrm{Cl}$ to make $\mathrm{Fe}(\mathrm{CN})_{6}{ }^{3-}$ catholyte and $\mathrm{Fe}(\mathrm{CN})_{6}{ }^{4-}$ and $\mathrm{Br}^{-}$anolytes, respectively. $\mathrm{CuSO}_{4}$ (Sigma-Aldrich, $\geq 99 \%$ ) was mixed with $\mathrm{H}_{2} \mathrm{SO}_{4}$ to make a $\mathrm{Cu}^{2+}$ catholyte (at $\mathrm{pH} 2$ ).

Sample preparation method. The silicon-based photoelectrodes used in this study were prepared using $\mathrm{n}$ - and p-type $\mathrm{CZ}$ c-Si wafers $(350 \mu \mathrm{m}$ thick, $1-20 \mathrm{Ohm} \mathrm{cm})$ Both $\mathrm{pn}^{+}$- and $\mathrm{np}^{+}$-junctions were formed using processes described in our previous publications ${ }^{1,16,17}$. Thin films of $\mathrm{Pt}(5-7 \mathrm{~nm})$ film was described in our previous publication (investigated by SEM, see Supplementary Fig. 10 in Supplementary Information). The carbon was prepared at room temperature with reactive sputter deposition on the back-side of the photoelectrodes. The deposition rate was calibrated with an in-chamber quartz crystal microbalance (QCM). Further, a ring- 
shaped Au layer was deposited on the light-illuminated surface using reactive sputtering with a home-made metallic mask at room temperature. For the electrochemical experiment under the dark, various conducting layers-including the $\mathrm{Pt}, \mathrm{C}$, and $\mathrm{TiO}_{2}-$ were deposited onto degenerately doped $\mathrm{n}^{+}-\mathrm{Si}$ substrates. The carbon was sputtered at room temperature or $400^{\circ} \mathrm{C}$. In the case of the $\mathrm{TiO}_{2}$, a thin layer of titanium was sputtered on first to prevent silicon oxidation in the subsequent $\mathrm{TiO}_{2}$ reactive sputtering step at $400^{\circ} \mathrm{C}$, as described elsewhere ${ }^{1}$.

Photo- and electrochemical measurements. Both PEC and electrochemical performances were characterized by using a PARSTAT MC potentiostat (Princeton Applied Research, AMETEK), $\mathrm{Hg} / \mathrm{HgSO}_{4}$ reference, and carbon-felt counter (Sigracet 39AA; $2 \mathrm{~cm}^{2}$ ) electrodes were used for all electrochemical measurements. The specific surface area of the carbon-felt is $\sim 0.5 \mathrm{~m}^{2} \mathrm{~g}^{-142}$. Calculation details for ECSA are provided in the Supporting Information section 8. The solar charging was done under simulated AM1.5 solar irradiation $\left(100 \mathrm{~mW} \mathrm{~cm}^{-2}\right)$ using a Newport Sol3A Class AAA solar simulator with a 450 W Xenon arc lamp. The solutions were purged with nitrogen before and during measurements. During the measurements, the electrolyte was continuously circulated using a peristaltic pump at $70 \mathrm{~mL} \mathrm{~min}^{-1}$

AFM, SEM, and thickness characterisation. Atomic force microscope (AFM) imaging was performed on the different samples presented in this work using a Bruker Dimension Icon atomic force microscope. A Bruker SCANASYST-AIR probe with force constant of $0.4 \mathrm{~N} / \mathrm{m}$ was used in the PeakForce Tapping ${ }^{\circledR}$ mode to obtain these images. As a supplementary tool, the thickness profilometer with a vertical resolution of 1 Å level was applied (Veeco Dektak 8 Surface Profiler). The SEM images were recorded using an FEI Verios 460 at an accelerating voltage of $10 \mathrm{kV}$.

Band alignment and theoretical efficiency calculation. The band alignment was calculated based on fundamental device physics, as shown in our previous works ${ }^{16,34}$. Simulated curves were obtained using the model, as described in our previous work ${ }^{17}$. Basically, the present work utilised the Bulter-Volmer equation for a single-electron reaction to account for the theoretical dependence of the kinetic overpotential on current density. Calculation details are provided in the Supporting Information section.

\section{Data availability}

The data that support the findings of this study are available within the paper and its supplementary information file.

\section{Code availability}

Python code that support the findings of this study have been deposited in TU Delft open access repository (http://resolver.tudelft.nl/uuid:8bc8ad4a-5aae-46cd-90a3-

bdac9c5432e0). It is also available from the corresponding authors upon reasonable request.

Received: 22 January 2020; Accepted: 13 March 2020;

Published online: 14 April 2020

\section{References}

1. Bae, D., Seger, B., Hansen, O., Vesborg, P. C. K. \& Chorkendorff, I. Durability testing of photoelectrochemical hydrogen production under day/night light cycled conditions. ChemElectroChem 6, 106-109 (2019).

2. Bae, D., Seger, B., Vesborg, P. C. K., Hansen, O. \& Chorkendorff, I. Strategies for stable water splitting: Via protected photoelectrodes. Chem. Soc. Rev. 46, 1933-1954 (2017).

3. Chu, S. et al. Photoelectrochemical $\mathrm{CO}_{2}$ reduction into syngas with the metal/ oxide interface. J. Am. Chem. Soc. 140, 7869-7877 (2018).

4. Wnag, L. et al. Greening ammonia toward the solar ammonia refinery. Joule $\mathbf{2}$, $1-20(2018)$.

5. Vesborg, P. C. K. \& Seger, B. Performance limits of photoelectrochemical $\mathrm{CO}_{2}$ reduction based on known electrocatalysts and the case for two-electron reduction products. Chem. Mater. 28, 8844-8850 (2016).

6. McKone, J. R., DiSalvo, F. J. \& Abruña, H. D. Solar energy conversion, storage, and release using an integrated solar-driven redox flow battery. J. Mater. Chem. A 5, 5362-5372 (2017).

7. Zhou, Y. et al. Efficient solar energy harvesting and storage through a robust photocatalyst driving reversible redox reactions. Adv. Mater. 30, 1-7 (2018).

8. Wang, Q., Sun, J. \& Chu, G. Lithium Ion Battery Fire And Explosion. Fire Safety Science 8, 375-382. https://doi.org/10.3801/IAFSS.FSS.8-375 (2005)

9. Kong, L., Li, C., Jiang, J. \& Pecht, M. G. Li-ion battery fire hazards and safety strategies. Energies 11, 1-11 (2018).
10. Beh, E. S. et al. A neutral $\mathrm{pH}$ aqueous organic/organometallic redox flow battery with extremely high capacity retention. ACS Energy Lett. 2, 639-644 (2017).

11. Li, L. et al. A stable vanadium redox-flow battery with high energy density for large-scale energy storage. Adv. Energy Mater 1, 394-400 (2011).

12. Luo, J. et al. A $1.51 \mathrm{vpH}$ neutral redox flow battery towards scalable energy storage. J. Mater. Chem. A 7, 9130-9136 (2019).

13. Hodes, G., Manassen, J. \& Cahen, D. Photoelectrochemical energy conversion and storage using polycrystalline chalcogenide electrodes. Nature 261, 403-404 (1976).

14. Wedege, K., Bae, D., Smith, W. A., Mendes, A. \& Bentien, A. Solar redox flow batteries with organic redox couples in aqueous electrolytes: a mini-review. $J$. Phys. Chem. C 122, 25729-25740 (2018).

15. Urbain, F. et al. Solar vanadium redox-flow battery powered by thin-film silicon photovoltaics for efficient photoelectrochemical energy storage. J. Phys. D. Appl. Phys. 52, 044001 (2019).

16. Wedege, $\mathrm{K}$. et al. Unbiased, complete solar charging of a neutral flow battery by a single Si photocathode. RSC Adv 8, 6331-6340 (2018).

17. Bae, D., Faasse, G. M., Kanellos, G. \& Smith, W. A. Unravelling the practical solar charging performance limits of redox flow battery based on the single photon device system. Sustain. Energy Fuels 3, 2399-2408 (2019).

18. Calderon, E. $\mathrm{H}$. et al. Effectiveness factor of fast $\left(\mathrm{Fe}_{3}+/ \mathrm{Fe}_{2}+\right)$, moderate $\left(\mathrm{Cl}_{2} /\right.$ $\mathrm{Cl}-)$ and slow $\left(\mathrm{O}_{2} / \mathrm{H}_{2} \mathrm{O}\right)$ redox couples using $\mathrm{IrO}_{2}$-based electrodes of different loading. J. Appl. Electrochem. 39, 1827-1833 (2009).

19. Cheng, Q. et al. Photorechargeable high voltage redox battery enabled by Ta3N5 and GaN/Si dual-photoelectrode. Adv. Mater. 29, 1-8 (2017).

20. Li, W., Fu, H. C., Zhao, Y., He, J. H. \& Jin, S. 14.1\% efficient monolithically integrated solar flow battery. Chem 4, 2644-2657 (2018).

21. Yu, Z. J., Carpenter, J. V. \& Holman, Z. C. Techno-economic viability of silicon-based tandem photovoltaic modules in the United States. Nat. Energy 3, 747-753 (2018)

22. Li, W. et al. Integrated photoelectrochemical solar energy conversion and organic redox flow battery devices. Angew. Chemie Int. Ed. 55, 13104-13108 (2016).

23. Liao, S. et al. Integrating a dual-silicon photoelectrochemical cell into a redox flow battery for unassisted photocharging. Nat. Commun. 7, 11474 (2016).

24. Flox, C. et al. Role of bismuth in the electrokinetics of silicon photocathodes for solar rechargeable vanadium redox flow batteries. ChemSusChem 11, $125-129$ (2018).

25. Harraz, F. A., Sasano, J., Sakka, T. \& Ogata, Y. H. Different behavior in immersion plating of nickel on porous silicon from acidic and alkaline fluoride media. J. Electrochem. Soc. 150, C277 (2003).

26. Thomas, J. H. Effect of pressure on dc planar magnetron sputtering of platinum. J. Vac. Sci. Technol. A Vacuum, Surfaces, Film 21, 572-576 (2003).

27. van der Rest, A. et al. Mechanical behavior of ultrathin sputter deposited porous amorphous $\mathrm{Al}_{2} \mathrm{O}_{3}$ films. Acta Mater. 125, 27-37 (2017).

28. Park, Y. S., Hong, B., Cho, S. J. \& Boo, J. H. Temperature dependence on structural, tribological, and electrical properties of sputtered conductive carbon thin films. Bull. Korean Chem. Soc 32, 939-942 (2011).

29. Mei, B. et al. Crystalline $\mathrm{TiO}_{2}$ : a generic and effective electron-conducting protection layer for photoanodes and -cathodes. J. Phys. Chem. C 119, 15019-15027 (2015).

30. McKone, J. R. et al. Evaluation of Pt, Ni, and Ni-Mo electrocatalysts for hydrogen evolution on crystalline Si electrodes. Energy Environ. Sci. 4, 3573 (2011).

31. Yao, T. et al. Manipulating the interfacial energetics of n-type silicon photoanode for efficient water oxidation. J. Am. Chem. Soc. 138, 13664-13672 (2016).

32. Lemishko, S. S. \& Lemishko, A. S. Cu2 $+/ \mathrm{Cu}+$ redox battery utilizing lowpotential external heat for recharge. J. Phys. Chem. C 121, 3234-3240 (2017).

33. Khataee, A., Wedege, K., Dražević, E. \& Bentien, A. Differential pH as a method for increasing cell potential in organic aqueous flow batteries. $J$. Mater. Chem. A 5, 21875-21882 (2017).

34. Bae, D. et al. Carrier-selective p- and n-contacts for efficient and stable photocatalytic water reduction. Catal. Today 290, 59-64 (2017).

35. Kemppainen, E. et al. Scalability and feasibility of photoelectrochemical H2 evolution: the ultimate limit of Pt nanoparticle as an HER catalyst. Energy Environ. Sci. 8, 2991-2999 (2015).

36. Shockley, W. \& Queisser, H. J. Detailed balance limit of efficiency of p-n junction solar cells. J. Appl. Phys. 32, 510-519 (1961).

37. Smith, W. A., Sharp, I. D., Strandwitz, N. C. \& Bisquert, J. Interfacial bandedge energetics for solar fuels production. Energy Environ. Sci. 8, 2851-2862 (2015).

38. Pourbaix, M. Atlas of Electrochemical Equilibria in Aqueous Solutions. (National Association of Corrosion Engineers, 1974).

39. Luo, J. et al. Bipolar membrane-assisted solar water splitting in optimal $\mathrm{pH}$. Adv. Energy Mater 6, 1-7 (2016). 
40. Durán Retamal, J. R., Periyanagounder, D., Ke, J. J., Tsai, M. L. \& He, J. H. Charge carrier injection and transport engineering in two-dimensional transition metal dichalcogenides. Chem. Sci 9, 7727-7745 (2018).

41. Pan, F. \& Wang, Q. Redox species of redox flow batteries: a review. Molecules 20, 20499-20517 (2015).

42. Forner-Cuenca, A., Penn, E. E., Oliveira, A. M. \& Brushett, F. R. Exploring the role of electrode microstructure on the performance of non-aqueous redox flow batteries. J. Electrochem. Soc. 166, A2230-A2241 (2019).

\section{Acknowledgements}

We gratefully acknowledge the financial supports provided by the LEaDing Fellowship from the European Union's Horizon 2020 research and innovation programme under the Marie Sklodowska-Curie grant agreement no. 707404. The authors also thank Dr. Kristina Wedege (Aarhus University) for assistance with TEMPO-sulfate synthesis, Joost Middelkoop, and Herman Schreuders at MECS group of TU Delft for assistance with electrochemical characterization and vacuum deposition system setup. The support of silicon substrates by the Dr. Choongman Moon (Technical University of Denmark) is also acknowledged.

\section{Author contributions}

D.B. designed the experiments, prepared the manuscript, and co-supervised G.K. and G.M.F. with W.A.S. G.K. performed the measurements of the electrochemical behaviours and characterization, and G.M.F. prepared theoretical calculation and modeling. A.V. provided the AFM measurement, and E.D. prepared TEMPO-sulfate chemical used for the redox reactions.

\section{Competing interests}

The authors declare no competing interests.

\section{Additional information}

Supplementary information is available for this paper at https://doi.org/10.1038/s43246020-0020-7.

Correspondence and requests for materials should be addressed to D.B. or W.A.S.

Reprints and permission information is available at http://www.nature.com/reprints

Publisher's note Springer Nature remains neutral with regard to jurisdictional claims in published maps and institutional affiliations.

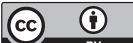

Open Access This article is licensed under a Creative Commons Attribution 4.0 International License, which permits use, sharing, adaptation, distribution and reproduction in any medium or format, as long as you give appropriate credit to the original author(s) and the source, provide a link to the Creative Commons license, and indicate if changes were made. The images or other third party material in this article are included in the article's Creative Commons license, unless indicated otherwise in a credit line to the material. If material is not included in the article's Creative Commons license and your intended use is not permitted by statutory regulation or exceeds the permitted use, you will need to obtain permission directly from the copyright holder. To view a copy of this license, visit http://creativecommons.org/ licenses/by/4.0/.

(C) The Author(s) 2020 